\title{
Status-quo of the Outlooks on Life of Medical Students in the Border Ethnic Areas and Effect of University Education on Them
}

\author{
Genliang Li $i^{a}$, Hui Qian ${ }^{b^{*}}$ \\ Basic Medical College, Youjiang Medical University for Nationalities, Baise, Guangxi 533000, The \\ People's Republic of China \\ aemail: ligenliang@163.com, ${ }^{\mathrm{b}}$ email: 2273006809@qq.com \\ * The corresponding author
}

Keywords: Border area; Ethnic minority; Medical college; Post-90s students; Outlook on life

\begin{abstract}
The research analyzed the roles of university education in outlook on life from the perspective of various university education units on the basis of questionnaire survey. 884 post-90s students of Youjiang Medical University for Nationalities, ethnic medical university in guangxi, in the border areas were used as statistic object in the study. Overall, those students' outlook on life is positive. Especially, they pay attention to both the individual pursuit of self improvement-and-development and the selfless dedication to society-and-community. However, the collegial education effects interrelated counld be forward improved. The effects of various university education units on the students' outlook on life are (extreme) different and those on the different subdimensions are various. Therefore, different education units and the inter-education-units can collaborate in the labour division of the education of outlook on life at the devierse subdimensions and play their respective advantages to maximize the education effect.
\end{abstract}

\section{Introduction}

The formation of correct outlook on life has a profound significance in cultivating and practicing social core values and the development of individuals and the whole society[1-5]. University period is the crucial one during which university students' outlooks on life are formed and the most significant stage when they are effectively conducted before they step into the society[4-6]. With the deepening of the reform and open policy and the high-speed development of society, contemporary undergraduates especially those students in minority areas are influenced by multi-culture more and more obviously, which make them a generation affected deeply by multi-culture[7-9]. The study analyzes the status-quo of the outlooks on life of post-90s medical students in the undeveloped border areas and the university education's effect on their outlook formation, so as to obtain possible educational enlightenment from the perspective of multi-level educational function units of universities.

\section{Objects and methods}

Research objects. Undergraduates of each grade in Youjiang Medical University for Nationalities were tested. A total of 1050 questionnaires were issued (Fig. 1). 923 of them were returned with a response rate of $87.9 \%$ and 884 of them were retrieved with an effective rate of $95.8 \%$.

Questionnaires. The research adopted the life cognitive scale which was compiled by Shi Lin and Li Qiong to study the university students' outlooks on life ${ }^{[10]}$.

Investigation methods. It refers to Liet al[1]. The test time was about 15 minutes. The data was collected in October, 2013.

Statistical process. All the statistics was processed by SPSS19.0. Two-factor or multi-factor analysis of variance as well as multiple comparisons were used to compare the significances of influence of different education background (undergraduate or specialist), length of schooling ( 3 years, 4 years or 5 years), majors 
(Clinical undergraduates, Nursing undergraduates, pharmacy undergraduates and pharmacy specialists), grades (grade1 to 4), classes (21 classes) and classroom teaching (biochemistry) on college students' outlooks on life. The significance level of statistics analysis was set as 0.05 .

\section{Results}

The overall situation of the college students' outlooks on Life. In terms of the overall level, the average scores of the five dimensions of the cognitive life scale for college students were all more than the middle value of 3 points $(n=884)$. The specific scores from high to low were as follows: the pursuit of self-improvement and self-development (SISD) $(3.98 \pm 0.47)(\bar{X} \pm \mathrm{s}$, hereinafter the same), the pursuit of services for the society and collectivity (SSC) $(3.93 \pm 0.40)$, the way of struggle (WS) $(3.55 \pm 0.49)$, the way of relaxation (WR) $(3.51 \pm 0.59)$ and the pursuit of utility and practicality (PUP) $(3.35 \pm 0.44)$. $n$ terms of average scores, college students got similar and the highest scores on the fractal dimensions of SISD and SSC, while they got the lowest scores on the fractal dimension of PUP. The results of two-factor multivariate analysis of variance showed that different factors had different influences on college students' outlooks on life. There was also significant difference in two-factor interactions ( $\mathrm{p}<0.05$, hereafter the same) or even extremely significant difference ( $\mathrm{p}<0.01$, hereafter the same) (Tab. 1 and Fig. 1). But no significant difference $(\mathrm{p}>0.05)$ was found in two factors such as educational background $\times$ length of schooling, education background $\times$ majors, educational background $\times$ grades, length of schooling $\times$ majors, length of schooling $\times$ grades, etc. The above results revealed that university education units had significant influence on college students' outlooks on life, but the effects of different fractal dimensions were different.

Table 1 Comparison of the outlooks on life of college students

\begin{tabular}{llllll}
\hline Outlooks on life & PUP & SISD & SSC & WS & WR \\
\hline$t_{\text {Gender }}$ & $4.65^{* *}$ & 0.12 & 0.55 & $2.37^{*}$ & 1.08 \\
$t_{\mathrm{EB}}$ & 1.19 & $3.50^{* *}$ & $2.69^{* *}$ & 0.08 & $3.22^{* *}$ \\
$F_{\mathrm{LS}}$ & 0.70 & $3.08^{* *}$ & 1.98 & 0.02 & $3.43^{* *}$ \\
$F_{\text {Gender } \times \text { LS }}$ & 0.87 & 0.62 & $3.18^{*}$ & 0.06 & 1.22 \\
$F_{\text {Majors }}$ & 2.17 & 1.52 & 0.70 & 0.44 & $10.15^{* *}$ \\
$F_{\text {Grades }}$ & $5.76^{* *}$ & $7.56^{* *}$ & $6.77^{* *}$ & 0.75 & 3.22 \\
$F_{\text {Classes }}$ & $2.96^{* *}$ & $2.24^{* *}$ & $2.24^{* *}$ & $2.05^{* *}$ & $1.91^{* *}$ \\
$F_{\text {Gender } \times \text { Classes }}$ & 1.30 & 1.29 & $1.59^{*}$ & 1.50 & $2.41^{* *}$ \\
$t_{\text {CT }}$ & 0.89 & $3.74^{* *}$ & 1.74 & 0.22 & 0.17 \\
$F_{\text {Gender } \times \text { CT }}$ & $5.18^{*}$ & 0.64 & 1.62 & 1.21 & 0.90 \\
\hline
\end{tabular}

Notes: ${ }^{*}$ Signific ant difference $(p<0.05) ;{ }^{* *}$ extremely significant difference $(p<0.01)$; EB, educ ational background; LS, length of schooling; CT, classroom teaching.

Comparison of the outlooks on life of college students of different gender, educational background and length of schooling. The average scores on five fractal dimensions of college students of different gender, educational background, and schooling length are showed in Fig. 1. The variance analysis indicated that boys and girls had statistical differences in utility $(p<0.01)$ and struggle $(p<0.05)$ (Fig. 1- I ). Students of junior colleges and undergraduate students had extremely significant differences in the following three fractal dimensions--perfection, society and relaxation (Fig. 1- II). Students of 3, 4 and 5 years of schooling length had extremely significant differences in dimensions of perfection and relaxation. The interaction effect of gender $\times$ length of schooling was significantly different in the dimension of society. The results of multiple comparison showed that there was significant difference in 3-4 and 3-5 years of schooling length on the dimension of perfection. On the dimension of relaxation, 3-4 and 3-5 years of length of schooling also had significant difference and extremely difference respectively (Fig. 1-III). The multiple comparison of two-factor 
interaction revealed that male students and female students with 4 years' length of schooling had significant difference in the dimension of society. The interactive effects of educational background, length of schooling and other factors were not significantly different in different dimensions.
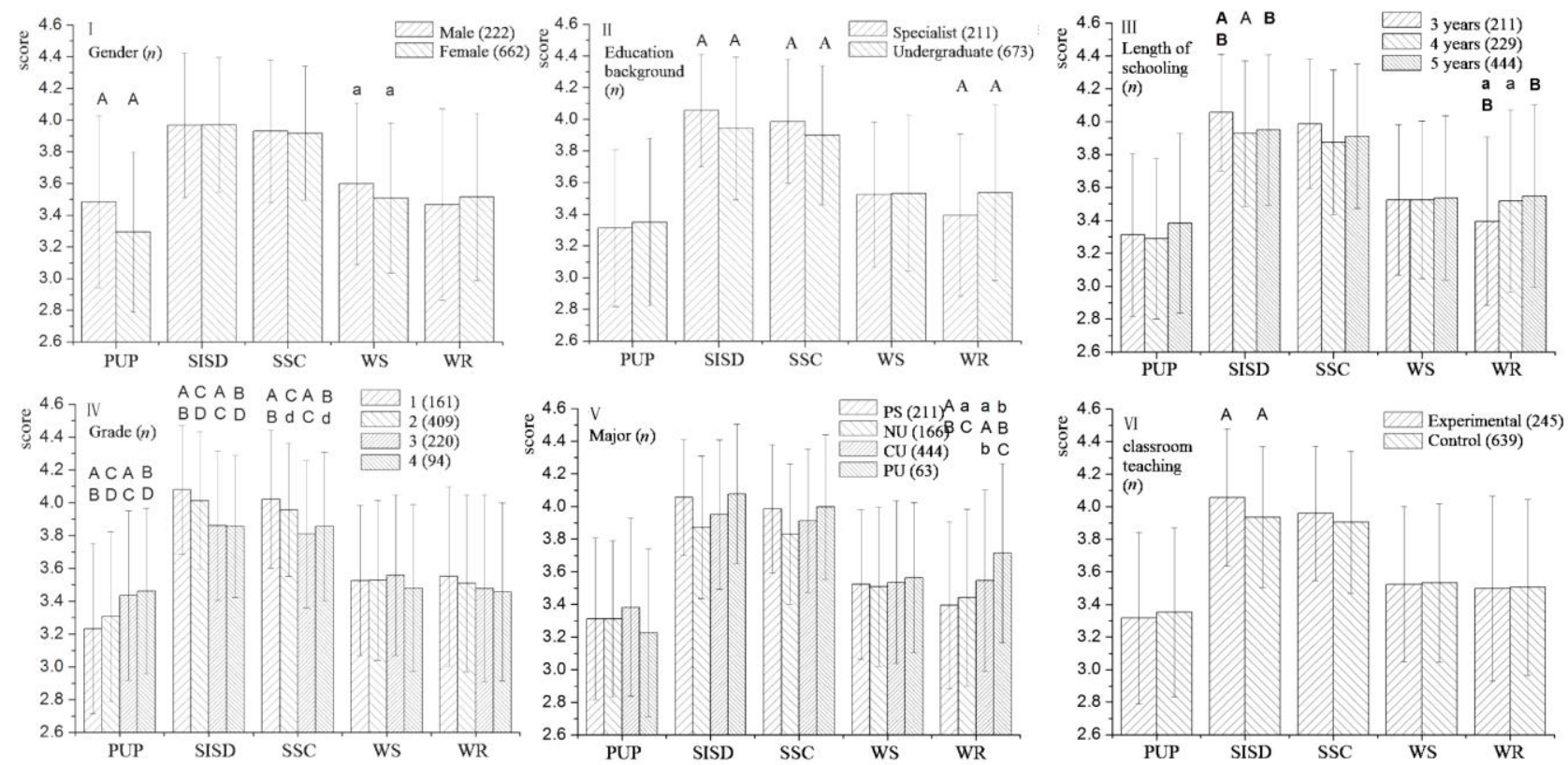

Fig. 1 The effects of various factors on the students' outlook on life $(\bar{X} \pm s, n=884)$. Identical lowercases on the bars in same group indicate significance of difference $(p<0.05)$ between each other; Identical majuscules on the bars in same group indicate extreme significance of difference $(p<0.01)$ between each other. PUP, pursuit of utility and practicality; SISD, self-improvement and self-development; SSC, services for the society and collectivity; WS, way of struggle; WR, way of relaxation.

Comparison of the outlooks on life of college students of different majors and grades. The average scores on five fractal dimensions of college students of different majors and grades were all over the median value 3.0; some of them were even more than 4.0 (Fig. 1-IV and 1- V). The scores on different dimensions of different grades were ranked from high to low as follows: Grade 4, 3, 2, 1 (PUP); Grade 1, 2, 3, 4 (SISD and WR) ; Grade 1, 2, 4, 3 (SSC) ; Grade 3, 2, 1, 4 (WS). Different majors got different scores (Fig. 1-IV). The results of the three-factor (gender, major and grade) variance analysis showed that different majors had only extremely significant difference on the WR. Nevertheless, different grades were extremely different on the PUP, SISD and SSC. The interactive effect of the two factors of gender $\times$ grade had significant differences in the SISD and extremely significant differences in both the PUP and the WR. The interactive effects of major $x$ grade factors had statistical differences in the three fractal dimensions of SSC $(p<0.05)$, WR $(p<0.05)$ and SISD $(p<0.01)$. The multiple comparison results showed that there was significant difference in the WR between clinical undergraduates and nursing undergraduates as well as between clinical undergraduates and pharmacy undergraduates while there were extremely significant differences between pharmacy specialists and clinical undergraduates, pharmacy specialists and pharmacy undergraduates, and between nursing undergraduates and pharmacy undergraduates in the WR (Fig. 1-V). There were an extremely significant differences between Grade 1 and Grade 3, Grade 1 and Grade 4, Grade 2 and Grade 3, and between Grade 2 and Grade 4 in the PUP and SISD; in the SSC, the difference between Grade 2 and Grade 4 was significant while those between Grade 1 and Grade 3, Grade 1 and Grade 4, and between Grade 2 and Grade 3 were extremely significant.

Comparison of the outlooks on life of college students in different classes. In terms of classes, the average scores on five fractal dimensions of college students were all different, from the lowest $(3.14 \pm 0.41)$ to the highest $(4.20 \pm 0.39)$. The variance analysis of the two factors of gender $\times$ class showed that: college 
students in different classes had significant differences on five different dimensions (Table 1). The interactive effect of the two factors of gender and class had significant difference on the SSC, especially on the WR.

Comparison of the outlooks on life of college students guided by teachers. Analysis of the two factors of gender and class indicated that classroom teaching also had significant influence on the outlooks on life of college students, which was mainly manifested on the SISD $(p<0.01)$. And the interactive effect of the two factors of genderxclass had influence on the dimension of PUP $(p<0.05)$ (Fig. 1-VI).

\section{Discussion}

The result of Yao ( and He)'s and Liu's investigation showed that post-80s and post-90s college students' outlook son life got the highest scores on the two fractal dimensions of perfection and society, to which our investigation result was similar ${ }^{[1,6]}$. As was showed in the investigation results, college students who were different in degrees, lengths of school, majors, grades and classes, and taught by different teachers had different outlooks on life. Compared with social and family education, university education has more powerful effect on guiding and correcting the outlooks on life of college students. The realization of this function relies on the university system of time and space, including multi-level educational units of space and time. The former includes the whole entity of university, secondary department (corresponding to majors), classes (corresponding to counselors and head teachers), classroom teaching (corresponding to course teachers) etc. The latter includes length of schooling (corresponding to expected duration of education), grades (corresponding to length of received education) etc ${ }^{[11]}$. Besides, university education also needs to take the differences of individuals into consideration, such as gender, education background (corresponding to the quality levels of enrolled student) etc. The longer education they get, the more attention to utility pursuing they pay, but the less consciousness of self-improvement and dedication to the social community they have.Results of the survey show that all factors above influence the education efficacy of university on students. From an overall perspective, most of students are positive, which indicates the education of outlooks on life in university is effective.

On the one hand, it suggests that college students tend to be realistic, rational and mature with the increase of experience; on the other hand, it also illustrates that less attention is paid to the education on the outlooks on life of college upperclassmen or the educational effect has been weakened, which leads to the weakening of college students' awareness of self-improvement and contribution to society. University education is more dispersed to various education units after a series of education activities at school. Therefore, it is necessary to integrate these education units in order to give full play to the guiding role of education unit in cultivating students' outlooks on life.

College students from different classes are extremely different in the five fractal dimensions of outlook on life, which signifies that the main managers (namely, counselors and head teachers) of classes play a tremendous role in building college students' outlooks on life. In various education units, counselors and head teachers have the greatest influence on college students' outlooks on life, indicating that counselors and head teachers must give full play in the education of college students' outlooks on life. This is one of the main territories for cultivating college students' outlooks on life, as well as one of the most effective ways.

Although the teaching period of course teachers for each class is relatively short, their intended instruction in the process of classroom teaching has a noticeable influence on the formation of college students' proper outlooks on life. Therefore, the education of college students' outlooks on life needs the full play of course teachers. In the process of classroom teaching, teachers should sufficiently integrate instructions of outlook on life into teaching content and actively guide the college students to cultivate proper outlooks on life and core socialist values. As Han Yu (an ancient Chinese educator) said, "A teacher is one who spreads truths, imparts professional knowledge, and resolves doubts", which told us that the primary function of teachers is to teach 
students how to behave themselves, and the second function is to teach them the skills of living and starting their career. But in the process of our current education, teachers seem to pay more attention to imparting knowledge instead of living and career skills, let alone teaching them how to behave themselves. Therefore, in the process of teaching, especially in college education, it is necessary to emphasize and give full play to the role of teachers in cultivating college students' outlooks on life.

Anyway, the values of ethnic medical college students in border areas are generally positive, but the function of college education on the outlooks on life of college students still needs to improve. Our college students particularly lay emphasis on self-improvement and selfless dedication to society and they also deal with the relationship between these two aspects very well. Each educational unit has its own effect focus on different fractal dimensions of college students' outlooks on life. The latter indicates that different educational units could cooperate with one another in their due divisions of labor on the disparate dimension of college students' outlook on life and exert their own superiorities so as to maximize the education effects. In addiction, multiple comparative results of different elements can be references for taking more effective teaching methods and measures to instruct different groups of college students, so that teaching could be better carried out in accordance of their aptitudes. The concrete measures of the integration of various education units and their optimizations are related to specific college and specific college student group, which remains to be further studied.

\section{Acknowledgements}

This study was funded by the Higher Education Teaching Reform Project of Guangxi Zhuang Autonomous Region, China (2013JGA203) and Innovation Project of Guangxi Zhuang Graduate Education (JGY2015136).

\section{References}

[1] G.L. Li, S. Nong, Analysis of socialist core values on individual level of Medical Students in the border ethnic areas, J. Youjiang Med. Coll. For National. 37(2015) 126-128, 134.

[2] M. Loughry, C. Eyber, Program on forced migration and health at the mailman school of public health, columbia universit, psychosocial concepts in humanitarian work with children: a review of the concepts and related literature, National Academies Press, Washington DC, 2003.

[3] H.J. Jeon, J.H. Park, E.J. Shim, Permissive attitude toward suicide and future intent in individuals with and without depression: results from a nationwide survey in Korea, J. Nerv. Ment. Dis. 201(2013) 286-291.

[4] K. Kato, R. Zweig, N. Barzilai, G. Atzmon, Positive attitude towards life and emotional expression as personality phenotypes for centenarians, Aging (Albany NY) 4(2012) 359-367.

[5] M. Nebhinani, N. Nebhinani, L. Tamphasana, A.D. Gaikwad, Nursing students' attitude towards suicide attempters: a study from rural part of Northern India, J Neurosci Rural Pract. 4(2013): 400-407.

[6] B.X. Yao, Y.Q He, A research on college students' outlook on life, Psycholog. Sci., 31(2008) 92, 97-99.

[7] B. Yao, Z.F. Peng, D.Y. Guo, Ideological and political education of college studentes from multicultural view, J. Hebei Univ. (Philosophy Social Sci.), 37(2012) 123-126.

[8] X. Bai, Y. Zhang, A study on ideological and polotical education of minoriy college students from the perspective of multicultural education concept, J. Hebei Univ. (Philosophy Social Sci.), 37(2012) 150-152.

[9] X.J. Wang, On college's cultivation and practice the socialist core value in the multi-cultural context, J. Continuing Higher Edu. 28(2015) 27-31. 
[10] L. Shi, Q. Li, The psychological study of Outlook on Life, Psycholog. Sci., 21(1998): 463-464.

[11] Y.H. Fu, F. Chen, New progress in family context influencing children's gender role socialization, J. Shanghai Norm. Univ.: Ele. Edu. Edi. 35(2006) 10-13. 